European Journal of Pain

\title{
STarT Back Tool risk stratification is associated with changes in movement profile and sensory discrimination in low back pain. A study of 290 patients. \\ --Manuscript Draft--
}

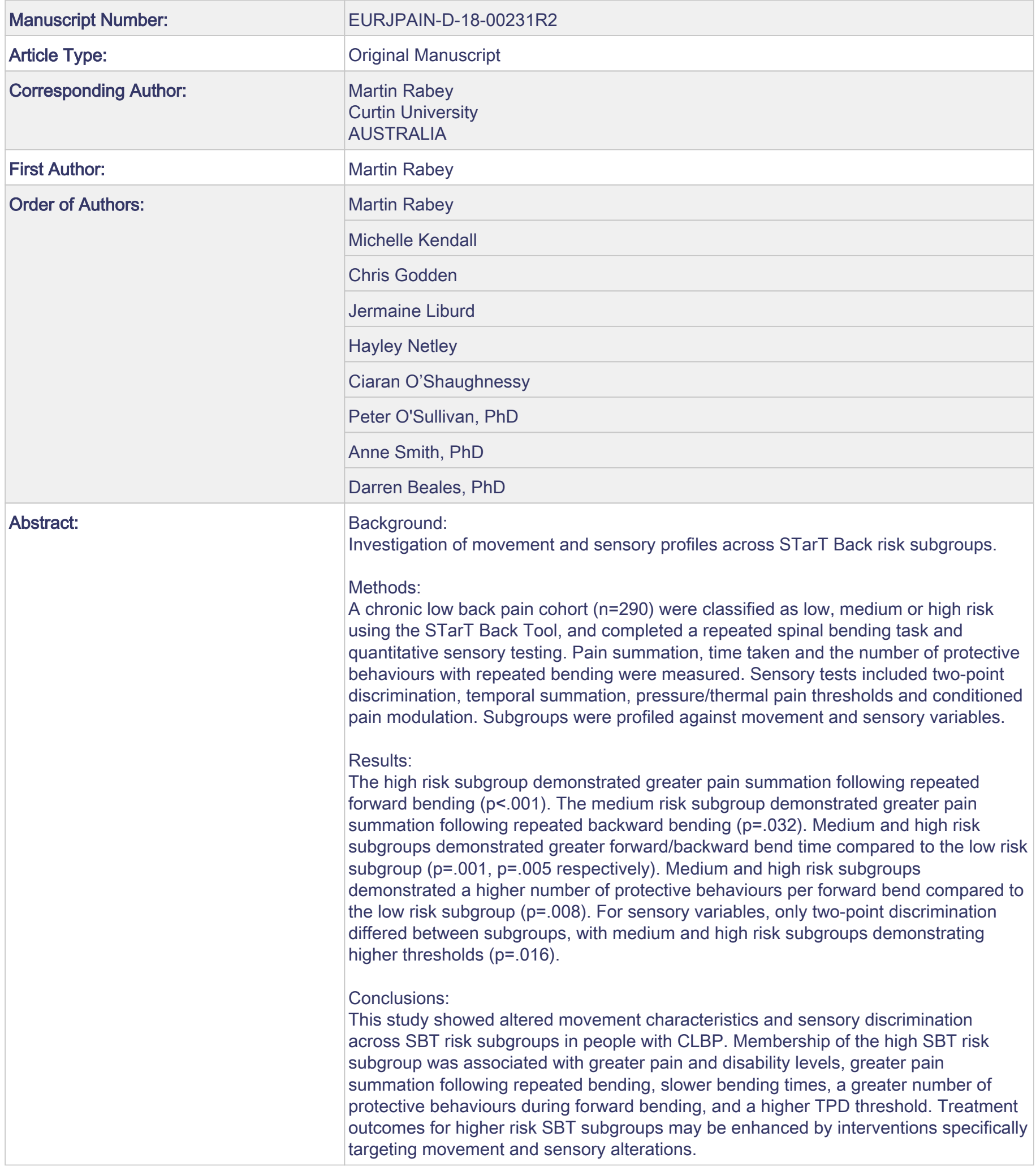




\section{Introduction}

Chronic low back pain (CLBP) is the leading cause of years lived with disability, with a point prevalence of 9.4\% (Hoy et al., 2014). Despite better understanding of the multidimensional nature of CLBP (Rusu et al., 2012) clinical outcomes are limited and healthcare expenditure high (Manchikanti et al., 2014; Vos et al., 2012). One approach to improve outcomes CLBP has been subgrouping (Costa et al., 2013; Foster et al., 2013).

The STarT Back Tool (SBT) was designed to stratify people with LBP into low, medium and high risk of future disability, with subgroups matched to care pathways (Hill et al., 2008). The effectiveness ofstratified care based on the SBT has been evaluated in in primary care (Hill et al., 2011). At 12-month follow-up stratified care had a small effect on disability, but no effect on pain intensity compared to controls (Hill et al., 2011). A broader understanding of the characteristics of SBT risk subgroups may facilitate targeting underlying mechanisms. Previous studies have demonstrated higher SBT risk subgroup allocation and/or score is associated with greater pain intensity (Field and Newell 2012; Fritz et al., 2011; Kendell et al., 2018), disability (Beneciuk et al., 2015; Field and Newell 2012; Fritz et al., 2011; Kendell et al., 2018; Pagé et al., 2015) and negative psychological affect and cognitions (Beneciuk et al., 2015; Kendell et al., 2018; Kongsted et al., 2011; Pagé et al., 2015) in keeping with constructs assessed by the tool. However, movement and sensory profiles of SBT risk subgroups have not been investigated.

Movement characteristics such as reduced movement speed, protective behaviours (guarding, bracing (Keefe and Block 1982)) and provocative pain following repeated movements (Rabey et al., 2017) have been associated with increased disability (Wong and Lee 2004) and negative psychological factors (Thomas et al., 2008) in people with CLBP.

Varying sensory profiles involving differences in thermal and pressure pain sensitivity, temporal summation and conditioned pain modulation (CPM) have been demonstrated in CLBP subgroups (Rabey et al., 2015; Scholz et al., 2009, Rabey et al. 2015, Coronado et al. 2014, O’Neill et al. 2014). While the relationship between sensory profile and pain or disability is unclear (Hübscher et al., 2013; Rabey et al., 2015), different sensory profiles may be associated with differing responses to interventions (Baron et al., 2012). Therefore, it may 
be important to consider movement and sensory profiles of SBT risk subgroups to assist refinement of stratified care.

The aim of this study was to investigate whether SBT risk subgroups in people with CLBP differed across movement and sensory variables. It was hypothesised: (i) higher SBT risk subgroup allocation would be associated with increased pain summation following repeated spinal bending, a higher proportion of participants with a bidirectional increase in pain following repeated spinal bending, slower bending time and more protective behaviours; (ii) higher SBT risk subgroup allocation would be associated with a higher two-point discrimination (TPD) threshold, enhanced temporal summation (TS), reduced pressure pain threshold (PPT), higher cold pain threshold (CPT), lower heat pain threshold (HPT) and reduced CPM. 


\section{Methods}

This research was conducted in accordance with the Declaration of Helsinki and was approved by the Human Ethics Research Committee at Curtin University (Approval Number: HRE2017-0658). All participants gave written informed consent.

\subsection{Participants}

Participants were recruited from private physiotherapy, psychology, and pain management clinics in Perth, Western Australia (WA) and via multimedia advertisements circulated throughout the community in metropolitan and regional WA.

Participants were aged 18-70 years with dominant axial non-specific LBP as indicated by $\geq$ $60 \%$ of the pain in the low back (between T12 and the gluteal fold) and $\leq 40 \%$ in the leg(s) (Wai et al., 2009), had pain of $\geq 3$-months duration with an average baseline pain intensity in the last week of $\geq 2$ on an 11-point Numerical Rating Scale (NRS) (Dworkin et al., 2005), and LBP-related disability of $\geq 5$ on the Roland Morris Disability Questionnaire (RMDQ) (Roland and Morris 1983). Exclusion criteria included an inability to understand English, pregnancy, diagnosed neurological disorder, presence of serious spinal pathology, spinal surgery in the last six months, or bilateral dorsal wrist or hand pain.

Prior publications involving this cohort have not considered the movement and sensory profiles of SBT risk subgroups (Kendell et al., 2018; Rabey et al., 2015; Rabey et al., 2017; Rabey et al., 2016).

\subsection{Variables}

\subsubsection{Demographics and Pain Characteristics}

Demographic variables included were age, sex, education level, employment status, occupation (manual or sedentary, based upon the Australian and New Zealand Standard Classification of Occupations (Australian Bureau of Statistics 2013)), compensation status as measured by a single question from the Fear Avoidance Beliefs Questionnaire (FABQ) (Waddell et al., 1993), pain intensity in the last week (11-point NRS) (Dworkin et al., 2005), LBP-related disability (RMDQ, scale 0-24 where higher scores equate to greater disability) (Roland and Morris 1983), pain duration, percentage of symptoms in the lumbar region as opposed to the leg (Wai et al., 2009), and overall general health in the previous two weeks as 
measured by a single question from the COOP-WONCA charts (scale 1-5 where lower scores indicate better health) (Van Weel et al., 2012). More detailed description is available elsewhere (Rabey et al., 2015; Rabey et al., 2017; Rabey et al., 2016).

\subsubsection{The STarT Back Tool}

The SBT is a nine item self-report tool summed to produce an overall score from zero to nine, and a psychological subscale score from zero to five. Higher scores indicate greater risk of poor disability outcome (Hill et al., 2008). Patients with an overall score of three points or less are stratified into the low risk subgroup. Patients who have an overall score of four or more points are stratified into either the medium risk or high risk subgroup via the psychological subscale score (subscale score of three points or less to the medium risk subgroup, or four or more points to the high risk subgroup). In a primary care cohort with low back pain of variable duration the SBT has acceptable to substantial discriminant validity and substantial reliability (Hill et al., 2008). The SBT has moderate predictive validity and acceptable discriminate ability for future disability in people with CLBP (Kendall et al., 2018).

\subsubsection{Movement variables}

Participants were asked to complete 20 forward bends followed by 20 backward bends using standardised instructions (Rabey et al., 2017). Participants were permitted to complete the tasks in whatever fashion they wanted at whatever speed they wanted. Prior to performing the first movement, a baseline rating of pain intensity (NRS) was established. With respect to forward bending, participants were asked to pick up a pencil off the ground with this counted as one forward bend. Placing the pencil back on the ground was counted as the second forward bend. With respect to backward bending, participants were asked to look at a marker placed on the ceiling approximately $60 \mathrm{~cm}$ behind them without turning around. During both tasks there was a brief pause every five repetitions for participants to rate their pain intensity. If participants did not complete the full 20 repetitions due to pain or fear of exacerbation of symptoms, the number of repetitions completed was recorded. The movements were recorded using two iPads (4th Generation) (Apple, California, USA) (1080p HD video recording) mounted on tripods. One iPad was placed directly in front of the participant, while the other was placed to provide a lateral view of the participant's lumbar spine region.

Pain summation following repeated forward and backward bending was quantified as the 
change in pain intensity as determined by subtracting the participant's score on a NRS after the last set of repetitions completed from the baseline score (Sullivan et al., 2009). Pain was only deemed to have increased if it had increased by the minimum clinically important difference (MCID) of two-points (Salaffi et al., 2004) on the NRS. Movement subgroups were formed by grouping participants into one of three subgroups: no increase in pain ( $<$ twopoint change in both directions); unidirectional increase in pain ( $\geq$ two-point change following either forward or backward bending); and bidirectional increase in pain ( $\geq$ twopoint change in both directions).

Forward and backward bending time, determined from video playback, was measured as time taken (seconds) to complete the first five forward or backward bends respectively. Bending time was measured from the initiation of the movement until the point at which the participant was standing erect again.

Protective behaviours were measured using a reliable method (Keefe and Block 1982) as a total count of behaviours observed on video playback. Behaviours included guarding (abnormally stiff or rigid movement), bracing (an extended limb supporting abnormal weight distribution), rubbing, or holding the affected area, as previously described (Keefe and Block 1982, p366). The mean number of protective behaviours per completed bend was established for both forward and backward bending.

\subsubsection{Sensory variables}

Two-point discrimination threshold was measured in centimetres and defined as the minimum distance between two caliper points at which the participant could say that they had been touched by two points rather than one. Testing occurred in the region of maximal lumbar pain using the method described by Moberg (1990) and updated by Luomajoki and Moseley (2011). Stimulation was applied in ascending and descending runs where the distance between the caliper points was increased or decreased by five millimetres at a time. A mean of three thresholds was used for analysis.

The first of several pain sensitivity variables was detection of enhanced TS. Testing, performed in the area of maximal lumbar pain using a $26 \mathrm{~g}$ nylon monofilament, involved 30 applications of the stimulus at $1 \mathrm{~Hz}$ (Scholz et al., 2009). Participants rated the pain intensity of the first and last stimuli on an NRS. Enhanced TS was deemed present if participants 
perceived the initial stimulus as non-noxious but it became noxious (increasing by $\geq$ twopoints on the NRS) during repeated stimulation, or if participants deemed the first stimulus as noxious and pain intensity increased by $\geq$ two-points on the NRS (Salaffi et al., 2004; Scholz et al., 2009).

Pressure $(\mathrm{kPa})$, cold and heat $\left({ }^{\circ} \mathrm{C}\right)$ pain thresholds (abbreviated as PPT, CPT, HPT respectively) were measured at the area of maximal lumbar pain and at the dorsal wrist joint line. PPT was recorded as the point at which the sensation of pressure changed to a sensation of pressure and pain (Rolke et al., 2006), and was measured using an algometer (probe size $1 \mathrm{~cm}^{2}$; Somedic AB, Sweden). Pressure was increased from $0 \mathrm{kPa}$, at $50 \mathrm{kPa} / \mathrm{s}$, until the participant pressed a button to indicate they had reached their PPT. Cold pain thresholdwas recorded as the point at which the sensation of cold became one of cold and pain (Rolke et al., 2006). and was measured using a Thermotest contact thermode (Somedic AB, Sweden; thermode contact area $2.5 \mathrm{~cm} \times 5 \mathrm{~cm}$ ). Testing began at $32^{\circ} \mathrm{C}$. The temperature of the thermode decreased by $1^{\circ} \mathrm{C} / \mathrm{s}$ until the participant detected their threshold and pressed a button or the device's lower temperature limit $\left(4^{\circ} \mathrm{C}\right)$ was reached. Heat pain threshold was measured using the same contact thermode and was recorded as the point at which the sensation of heat became one of heat and pain (Rolke et al., 2006). Testing began at $32^{\circ} \mathrm{C}$. The temperature increased by $1{ }^{\circ} \mathrm{C} / \mathrm{s}$ until the participant detected their threshold and pressed a button or the device's upper temperature limit $\left(50^{\circ} \mathrm{C}\right)$ was reached. The mean of three thresholds at each site for each stimulus was used for analysis. Thirty-second inter-stimulus intervals were adopted to reduce the possibility of temporal summation.

To assess CPM, an algometer was used to apply pressure at the area of maximal lumbar pain starting at $0 \mathrm{kPa}$ and increasing at $50 \mathrm{kPa} / \mathrm{s}$ until the participant indicated that the pressure was equal to a pain intensity of $6 / 10$ on the NRS by triggering the algometer button (test stimulus). This pressure was recorded as the baseline CPM pressure. Noxious heat (conditioning stimulus) was then applied using the Thermotest to the dorsum of the hand. The conditioning stimulus began at $40{ }^{\circ} \mathrm{C}$ and was increased by one degree at a time until the participant indicated that the stimulus was too uncomfortable to tolerate, at which time it was reduced by one degree. Specific instructions were then given to the participant: "I am going 
to apply three further pressure tests to your back. You rated the last pressure as 6/10. Now I want you to give me a score out of ten compared to that first rating for each of these next three pressures." The test stimulus was then reapplied in the same way, at the same point, at the same application rate, to the predetermined pressure indicated by the participant $( \pm 10$ $\mathrm{kPa}$ ). The participant was asked to indicate the pain intensity (NRS) of the test stimuli while the conditioning stimulus was ongoing. The test stimuli were tested during the conditioning stimulus at three time points: 30,60 , and 90 seconds. The mean of the NRS scores from each time point were subtracted from the baseline NRS score $(6 / 10)$ and considered the CPM change score for analysis.

\subsection{Data analysis}

Descriptive statistics were calculated for the demographic variables, pain intensity and disability, and the movement and sensory variables with respect to the total cohort and each SBT risk subgroup. Subgroup differences for continuous demographic, pain intensity, disability, movement and sensory variables were examined using a one-way analysis of variance (ANOVA) for normally distributed variables and the Kruskal-Wallis test for variables with non-normally distributed data. Subgroup differences for categorical variables were examined using the chi-squared test. Potential confounding by gender of differences in pain sensitivity measures across SBT risk groups was tested by either tobit regression (CPTs and HPTs), logistic regression (enhanced TS) or linear regression with bootstrapped standard errors (all other variables). No corrections were made for multiple comparisons. With the sample size of 290 and the observed risk subgroup proportions, this study was adequately powered to detect meaningful differences in variables between risk subgroups, having a power of 0.8 to detect standardised mean differences as small as 0.4 at $\alpha=.05$. All data analyses were performed using Stata 13.1 (Statacorp, Texas, USA). Statistical significance was $\mathrm{p}<0.05$ for all analyses.

\section{Results}

\subsection{Participant demographics}

A total of 290 participants were included in the study with 228 (78.6\%) participants recruited from the general community, 59 (20.3\%) from private physiotherapy clinics, and three $(1.0 \%)$ from psychology and pain management clinics. Participant characteristics are presented in Table 1. The SBT stratified 82 participants $(28.3 \%)$ as low risk, $116(40.0 \%)$ as medium risk, and $92(31.7 \%)$ as high risk. With respect to the demographic variables, the SBT risk 
subgroups only differed for education level $(\mathrm{p}=0.01)$, occupation $(\mathrm{p}<0.001)$, and overall general health $(\mathrm{p}<0.001)$. The high risk subgroup had significantly fewer years in education than the low risk subgroup and the highest proportion of participants with manual jobs. General health worsened in a stepwise manner from the low risk subgroup to the high risk subgroup. The high risk subgroup had a lower proportion of females but this was not a statistically significant difference. Pain intensity and disability were significantly different across the SBT risk subgroups $(\mathrm{p}<0.001)$ with pain and disability increasing stepwise from the low through to the high risk subgroup.

\section{INSERT TABLE 1 HERE}

\subsection{Movement variables}

There were significant differences between SBT risk subgroups for all movement variables except the mean number of protective behaviours per backward bend completed (Table 2). Group contrasts indicated the high risk subgroup demonstrated greater pain summation following repeated forward bending than both the low risk subgroup(difference in median +1.5 ) and the medium risk subgroup (difference in median +1.0 ). The medium risk subgroup demonstrated greater pain summation following repeated backward bending than both the low risk subgroup (difference in median +1.0 ) and the high risk subgroup (difference in median +1.0 ). There was a significant difference between SBT risk subgroups in terms of membership of movement subgroups ( $\mathrm{p}=0.028$ ), with a higher than expected proportion of participants with no increase in pain in the low risk subgroup (63.4\%) and a higher than expected proportion of participants with a unidirectional (45.7\%) or a bidirectional $(15.2 \%)$ increase in pain in the high risk subgroup. The low risk subgroup had significantly faster forward bending times than the medium risk subgroup (difference in median -2.0secs) and the high risk subgroup (difference in median $-3.0 \mathrm{secs}$ ), and also significantly faster backward bending times than the medium and high risk subgroups (difference in medians $-1.5 \mathrm{secs}$ for both). Lastly, although the median protective behaviours per forward bend were the same across subgroups, the rank mean was significantly lower for the low risk subgroup (123) than the than the medium risk subgroup $(150, \mathrm{p}=0.014)$ and the high risk subgroup $(159, \mathrm{p}=0.002)$.

\section{INSERT TABLE 2 HERE}

\subsection{Sensory variables}


The SBT risk subgroups did not differ with respect to all but one pain sensitivity variable (Table 3) and regression analyses confirmed results were not confounded by the slight gender imbalance across risk subgroups. TPD threshold was different between groups, with the low risk subgroup having a significantly lower TPD threshold than the medium risk subgroup (gender adjusted mean difference $=-0.7,95 \%$ Confidence Interval $0.1-1.3, p=0.022$ ) and the high risk subgroup (gender adjusted mean difference $=-0.8,95 \%$ Confidence Interval 0.1 $1.4, \mathrm{p}=0.016)$.

\section{INSERT TABLE 3 HERE}

\section{Discussion}

This study profiled movement and sensory characteristics of SBT risk subgroups in people with CLBP. Regarding the first hypothesis, movement profiles of SBT risk subgroups broadly differed. Contrastingly, the second hypothesis was largely unsupported as there was no difference in sensory profiles of risk subgroups except TPD threshold.

\subsection{Movement variables}

Following repeated (forward) spinal bending, the high risk subgroup demonstrated greater pain summation, were more likely to experience unidirectional/bidirectional increases in pain, had slower bending times and exhibited greater protective behaviours. The high risk subgroup is defined by higher psychological distress. Prior investigation in this cohort reported a weak association between psychological distress and pain summation following repeated spinal bending (Rabey et al., 2017; Rabey et al., 2016), consistent with results reported here.

Possibly the only other study to have investigated the relationship between repeated movements and psychological factors found higher fear of movement, pain catastrophizing, and depression to be associated with greater pain summation with repeated lifting (Sullivan et al., 2009). However, the authors made no attempts to derive subgroups. In this current study the high risk subgroup had a greater proportion of manual workers, and a lower level of education. Greater pain summation and protective behaviours following repeated bending may have been influenced by work-related spinal loading (Coenen et al., 2014; Hayden et al., 2009).Conversely, prior investigation in this cohort reported better cognitive and affective psychological questionnaire scores in a people with no increase in pain following repeated spinal bending (Rabey et al., 2017). This is consistent with the present study where there was 
a higher than expected proportion of participants with no increase in pain in the low risk subgroup defined by no or low psychological distress.

Pain summation following backward bending did not conform to the above generalisation, with the medium risk subgroup demonstrating greater summation than others. Underlying forward bending movement characteristics may be the influence of implicit (Caneiro et al., 2017) and conscious (Darlow et al., 2014) beliefs related to harm/threat. These beliefs may differ for backwards bending, contributing to different findings for this movement across subgroups.

Forty-nine percent of participants showed no increase in pain with repeated bending. This may reflect the stipulation in this study for pain intensity to have changed by at least twopoints to be considered increased, the variability of pain responses to movement across the cohort, and that pain is often reported to improve following repeated movements (May and Aina, 2012).

Protective behaviours are considered maladaptive responses to feared, threatening and/or painful activities reflecting a person's attempt to enhance their perception of control and safety (O’Sullivan et al., 2018). Slower bending times and greater protective behaviours exhibited by the high risk subgroup may be influenced by greater pain summation (Hodges and Smeets 2015) or altered muscle activity with repeated movement (Falla et al., 2014). Greater pain intensity and disability have been associated with greater protective behaviours during repeated lifting (Sullivan et al., 2006). Higher fear-avoidance beliefs in people with LBP have also been associated with decreased peak velocity and acceleration of lumbar movement (Thomas et al., 2008). While fear was not an isolated construct investigated in the present study, it is a domain within the SBT and results from these studies investigating fear and movement align to our findings.

No previous studies have examined differences in movement characteristics across SBT risk subgroups. These findings suggest pain responses following repeated movement belie a complex relationship between movement and pain where changes in movement are possibly influenced by psychological, behavioral and pain-related factors. This may be both cause and effect of nociceptive input and/or pain (Hodges and Smeets 2015) (e.g. fear may lead to altered movement patterns which may influence tissue loading and subsequent peripheral 
nociceptive input). Interventions could be developed to target movement alterations, and associated psychological factors, for the high risk subgroup in particular, possibly affording improved treatment outcomes.

\subsection{Sensory variables}

Two-point discrimination threshold was the only sensory variable that differed across risk subgroups, being significantly lower in the low risk subgroup . Increased TPD threshold is considered to reflect greater disruption of body schema in the primary somatosensory cortex (Pleger et al., 2006). Increased TPD threshold has been associated with altered perception of body image and poorer lumbar spine motor control in people with CLBP (Luomajoki and Moseley 2011; Nishigami et al., 2015). While speculative, higher TPD thresholds reflecting cortical alterations in the medium and high risk subgroups, may adversely influence movement patterns and perception of the low back region, contributing to peripheral nociception secondary to altered mechanical loading (Hodges and Smeets 2015) and/or "topdown" nociceptive facilitation (Wand et al., 2016).

Pain sensitivity did not differ across SBT risk subgroups. The SBT was developed to predict poor disability outcome in people with LBP attending primary care (Hill et al. 2008) and predicted persistent disability in this cohort with CLBP (Kendall et al. 2018). However, consistent with recent reviews, there does not appear to be a clear association between pain and disability with quantitative sensory testing (QST) findings in people with LBP (Hübscher et al., 2013), and QST findings may not be prognostic of pain intensity or work absenteeism for people with LBP (Marcuzzi et al., 2016). Therefore, while the SBT stratifies people based upon risk of poor disability outcome, disability levels may not be influenced by their pain sensitivity. The mean CPM change score (1.0) in this study equates to a $17 \%$ change. CPM effects from widely varying protocols vary between 5-100\% (Pud et al., 2009). A 17\% change is toward the lower end of this range, however, it is unknown whether a different CPM protocol may have given different results and there is currently insufficient data to support one optimal CPM protocol (Yarnitsky et al., 2010). While speculative, examining pain sensitivity features may be relevant in clinical examination at the individual level, to understand possible pain mechanisms and inform treatment choices (Uddin and Macdermid 2016). While there were no significant differences between SBT subgroups for sex, the high risk subgroup tended towards a higher proportion of males. As females consistently have 
higher pain sensitivity (Mogil, 2012) this imbalance could have introduced potential bias. However, statistical analyses of sensory measures adjusting for gender confirmed this was not the case.

\subsection{Clinical implications}

The SBT was developed for use in primary care $(60 \%$ of participants had symptoms longer than three-months duration) (Hill et al., 2008), however, it has been shown to have adequate predictive ability for future disability in this CLBP cohort (Kendall et al., 2018) so may be an appropriate screening tool in such populations. Since chronic pain and disability levels may be responsive to intervention (Saragiotto et al., 2017) the movement and sensory findings from this study may inform refinement of stratified care. Pain and disability outcomes for the medium and high risk subgroup might be enhanced via management approaches which address alterations in movement and tactile acuity and appreciate their interconnectedness with negative psychological affect and cognitions. For example, in people with CLBP stratified by the SBT to medium or high risk subgroups a combination of pain education, sensory acuity re-training and movement retraining yielded significant reductions in pain intensity (Walti et al., 2015). Improvements in pain and disability have also been shown in people with CLBP following an intervention combining education and cognitive restructuring, specific movement and body schema retraining and lifestyle interventions (Vibe Fersum et al., 2013). Lifestyle factors may be important to consider in the management of people with poorer general health (Hartvigsen et al., 2013), as reported by the higher risk subgroups in this study. Such interventions have not been tested in acute or primary care settings, however, evaluation and subsequent targeting of movement and sensory alterations at that stage may reduce chronicity.

\subsection{Strengths and limitations}

Strengths of this study include the large sample size and inclusion of a broad range of potentially modifiable movement and sensory variables easily evaluated in a clinical setting. Generalisability of these results may be limited to CLBP populations with similar demographics in terms of dominant axial LBP rather than dominant leg pain, long duration of symptoms, moderate pain intensity, and relatively low LBP-related disability.

The SBT is only one of many systems for deriving subgroups of people with LBP (see Fairbank et al. (2011) for further details). In this and a separate cohort with CLBP it has moderate predictive ability for disability, however, its predictive ability for pain and global 
perceived change are comparatively poor (Kendall et al., 2018, Page et al., 2015). The SBT was developed to predict future disability (Hill et al., 2008) which may explain its poor performance regarding these other outcomes. CLBP is a multidimensional disorder (Rusu et al., 2012), however, the SBT lacks consideration of numerous dimensions associated with CLBP (e.g. lifestyle, general health) as well as the pain sensitivity and movement dimensions examined in this study. Also, changes in SBT risk subgroup allocation can occur in a short period of time (Newell et al., 2015), indicating the fluctuating nature of different factors underlying CLBP. Stratified care based upon SBT risk subgroups has not yet been examined in people with CLBP, however, to facilitate optimal treatment outcomes it may be important for management to target all relevant dimensions in a person's presentation (Linton et al., 2018).

\subsection{Conclusion}

This study showed different movement and sensory characteristics of SBT risk subgroups in people with CLBP. Membership of the high risk subgroup was associated with greater pain and disability levels, greater pain summation with repeated forward bending, slower bending times, greater protective behaviours during forward bending, and a higher TPD threshold. The modest outcomes shown for stratified care (Hill et al., 2011) may be enhanced for the high risk subgroup if interventions specifically targeted associated movement and sensory alterations. 


\section{References}

Australian Bureau of Statistics. 1220.0 - ANZSCO -- Australian and New Zealand Standard Classification of Occupations, 2013, Version 1.2 2013; Available from: http://www.abs.gov.au/ausstats/abs@.nsf/mf/1220.0.

Baron R, Förster M, Binder A. Subgrouping of patients with neuropathic pain according to pain-related sensory abnormalities: a first step to a stratified treatment approach. The Lancet Neurology 2012;11: 999-1005.

Beneciuk JM, Robinson ME, George SZ. Subgrouping for Patients With Low Back Pain: A Multidimensional Approach Incorporating Cluster Analysis and the STarT Back Screening Tool. The Journal of Pain 2015;16: 19-30.

Caneiro J, O’Sullivan P, Smith A, Moseley G, Lipp O. Implicit evaluations and physiological threat responses in people withpersistent low back pain and fear of bending. Scandinavian Journal of Pain 2017; 17: 355-366.

Coenen P, Kingma I, Boot C, Bongers P, van Dieën J. Cumulative mechanical low-back load at work is a determinant of low-back pain. Occupational and Environmental Medicine 2014; 71:332-337.

Coronado RA, Bialosky JE, Robinson ME, George SZ. Pain Sensitivity Subgroups in Individuals With Spine Pain: Potential Relevance to Short-Term Clinical Outcome. Physical Therapy 2014;94: 1111-1122.

Costa L, Koes BW, Pransky G, Borkan J, Maher C, Smeets R. Primary Care Research Priorities in Low Back Pain. An Update. Spine 2013;38: 148-156.

Darlow B, Perry M, Stanley J, Mathieson F, Melloh M, Baxter GD, Dowell A. Crosssectional survey of attitudes and beliefs about back pain in New Zealand. BMJ Open $2014 ; 4$.

Dworkin RH, Turk DC, Farrar JT, Haythornthwaite JA, Jensen MP, Katz NP, Kerns RD, Stucki G, Allen RR, Bellamy N, Carr DB, Chandler J, Cowan P, Dionne R, Galer BS, Hertz S, Jadad AR, Kramer LD, Manning DC, Martin S, McCormick CG, McDermott MP, McGrath P, Quessy S, Rappaport BA, Robbins W, Robinson JP, Rothman M, Royal MA, Simon L, Stauffer JW, Stein W, Tollett J, Wernicke J, Witter J. Core outcome measures for chronic pain clinical trials: IMMPACT recommendations. PAIN 2005;113: 9-19.

Fairbank J, Gwilym S, France J, Daffner S, Dettori J, Hermsmeyer J, Andersson G. The Role of Classification of Chronic Low Back Pain. Spine 2011;36: S19-S42.

Falla D, Gizzi L, Tschapek M, Erlenwein J, Petzke F. Reduced task-induced variations in the distribution of activity across back muscle regions in individuals with low back pain. Pain 2014;155: 944-953.

Field J and Newell D. Relationship between STarTBack Screening Tool and prognosis for low back pain patients receiving spinal manipulative therapy. Chiropr Manual Ther 2012;20: 17.

Foster NE, Hill JC, O'Sullivan P, Hancock M. Stratified models of care. Best Practice \& Research Clinical Rheumatology 2013;27: 649-661.

Fritz J, Beneciuk J, George S, Fritz J, Beneciuk J, George S. Relationship between categorization with the STarTBack screening tool and prognosis for people receiving physical therapy for low back pain. Phys Ther 2011;91: 722 - 732.

Hartvigsen J, Natvig B, Ferreira M. Is it all about a pain in the back? Best Practice \& Research Clinical Rheumatology 2013;27: 613-623.

Hayden J, Chou R, Hogg-Johnson S, Bombardier C. Systematic reviews of low back pain prognosis had variable methods and results - guidance for future prognosis reviews. Journal of Clinical Epidemiology 2009;62: 781-796. 
Hill J, Dunn K, Lewis M, Mullis R, Main C, Foster N, Hay E. A primary care back pain screening tool: identifying patient subgroups for initial treatment. Arthritis Rheum 2008;59: 632 - 641.

Hill JC, Whitehurst DGT, Lewis M, Bryan S, Dunn KM, Foster NE, Konstantinou K, Main CJ, Mason E, Somerville S, Sowden G, Vohora K, Hay EM. Comparison of stratified primary care management for low back pain with current best practice (STarT Back): a randomised controlled trial. The Lancet 2011;378: 1560-1571.

Hodges P and Smeets R. Interaction Between Pain, Movement, and Physical Activity: Shortterm Benefits, Long-term Consequences, and Targets for Treatment. Clinical Journal of Pain 2015;31: 97-107.

Hoy D, March L, Brooks P, Blyth F, Woolf A, Bain C, Williams G, Smith E, Vos T, Barendregt J, Murray C, Burstein R, Buchbinder R. The global burden of low back pain: estimates from the Global Burden of Disease 2010 study. Annals of the Rheumatic Diseases 2014;73: 968-974.

Hübscher M, Moloney N, Leaver A, Rebbeck T, McAuley JH, Refshauge KM. Relationship between quantitative sensory testing and pain or disability in people with spinal pain - A systematic review and meta-analysis. PAIN 2013;154: 1497-1504.

Keefe FJ and Block AR. Development of an observation method for assessing pain behavior in chronic low back pain patients. Behavior Therapy 1982;13: 363-375.

Kendell M, Beales D, O'Sullivan P, Rabey M, Hill J, Smith A. The predictive ability of the STarT Back Tool was limited in people with chronic low back pain: a prospective cohort study. Journal of Physiotherapy 2018;64: 107-113.

Kongsted A, Johannesen E, de Leboeuf Y. Feasibility of the STarT back screening tool in chiropractic clinics: a cross sectional study of patients with low back pain. Chiropr Manual Ther 2011;19: 10.

Linton S, Nicholas M, Shaw W. Why wait to address high risk cases of acute low back pain? A comparison of stepped, stratified and matched care. Pain 2018; In press.

Luomajoki $\mathrm{H}$ and Moseley GL. Tactile acuity and lumbopelvic motor control in patients with back pain and healthy controls. British Journal of Sports Medicine 2011;45: 437-440.

Manchikanti L, Singh V, Falco FJE, Benyamin RM, Hirsch JA. Epidemiology of Low Back Pain in Adults. Neuromodulation: Technology at the Neural Interface 2014;17: 3-10.

Marcuzzi A, Dean C, Wrigley P, Chakiath R, Hush J. Prognostic value of quantitative sensory testing in low back pain: a systematic review of the literature. Journal of Pain Research 2016;9: 599-607.

May, S. \& Aina, A. Centralization and directional preference: A systematic review. Manual Therapy 2012;17: 497-506.

Moberg E. Two point discrimination test. A valuable part of hand surgical rehabilitation. Scandinavian Journal of Rehabilitation Medicine 1990;22: 127-134.

Mogil, J. S. Sex differences in pain and pain inhibition: multiple explanations of a controversial phenomenon. Nature Reviews. Neuroscience 2012;13: 859-66.

Newell D, Field J, Pollard D. Using the STarT Back Tool: Does timing of stratification matter? Manual Therapy. 2015;20: 533-9.

Nishigami T, Mibu A, Osumi M, Son K, Yamamoto S, Kajiwara S, Tanaka K, Matsuya A, Tanabe A. Are tactile acuity and clinical symptoms related to differences in perceived body image in patients with chronic nonspecific lower back pain? Manual Therapy 2015;20: 63-67.

O`Neill S, Manniche C, Graven-Nielsen T, Arendt-Nielsen L. Association between a Composite Score of Pain Sensitivity and Clinical Parameters in Low-Back Pain. Clinical Journal of Pain 2014;30: 831-838. 
O’Sullivan PB, Caneiro JP, O'Keeffe M, Smith A, Dankaerts W, Fersum K, O’Sullivan K. Cognitive Functional Therapy: An Integrated Behavioral Approach for the Targeted Management of Disabling Low Back Pain. Physical Therapy 2018;98: 408-423.

Pagé I, Abboud J, O’Shaughnessy J, Laurencelle L, Descarreaux M. Chronic low back pain clinical outcomes present higher associations with the STarT Back Screening Tool than with physiologic measures: a 12-month cohort study. BMC Musculoskeletal Disorders 2015;16: 201.

Pleger B, Ragert P, Schwenkreis P, Förster A-F, Wilimzig C, Dinse H, Nicolas V, Maier C, Tegenthoff M. Patterns of cortical reorganization parallel impaired tactile discrimination and pain intensity in complex regional pain syndrome. NeuroImage 2006;32: 503-510.

Pud, D., Granovsky, Y. \& Yarnitsky, D. The methodology of experimentally induced diffuse noxious inhibitory control (DNIC)-like effect in humans. Pain 2009;144: 16-19.

Rabey M, Poon C, Wray J, Thamajaree C, East R, Slater H. Pro-nociceptive and antinociceptive effects of a conditioned pain modulation protocol in participants with chronic low back pain and healthy control subjects. Manual Therapy 2015;20: 763768.

Rabey M, Slater H, O'Sullivan P, Beales D, Smith A. Somatosensory nociceptive characteristics differentiate subgroups in people with chronic low back pain: a cluster analysis. Pain 2015;156: 1874-1884.

Rabey M, Smith A, Beales D, Slater H, O'Sullivan P. Pain provocation following sagittal plane repeated movements in people with chronic low back pain: Associations with pain sensitivity and psychological profiles. Scandinavian Journal of Pain 2017; 16: 2228.

Rabey M, Smith A, Beales D, Slater H, O'Sullivan P. Differing psychologically-derived clusters in people with chronic low back pain are associated with different multidimensional profiles. . Clinical Journal of Pain 2016;32: 1015-1027.

Roland M and Morris R. A study of the natural history of back pain. Part I: Development of a reliable and sensitive measure of disability in low-back pain. Spine 1983;8: 141144.

Rolke R, Baron R, Maier C, Tölle TR, Treede RD, Beyer A, Binder A, Birbaumer N, Birklein F, Bötefür IC, Braune S, Flor H, Huge V, Klug R, Landwehrmeyer GB, Magerl W, Maihöfner C, Rolko C, Schaub C, Scherens A, Sprenger T, Valet M, Wasserka B. Quantitative sensory testing in the German Research Network on Neuropathic Pain (DFNS): Standardized protocol and reference values. PAIN 2006;123: 231-243.

Rusu A, Boersma K, Turk D. Reviewing the Concept of Subgroups in Subacuteand Chronic Pain and the Potential of Customizing Treatments. In: From Acute To Chronic Back Pain : Risk Factors, Mechanisms, And Clinical Implications.Oxford: Oxford University Press; 2012; 485-511.

Salaffi F, Stancati A, Silvestri CA, Ciapetti A, Grassi W. Minimal clinically important changes in chronic musculoskeletal pain intensity measured on a numerical rating scale. European Journal of Pain 2004;8: 283-291.

Saragiotto B, Maher C, Traeger A, Li Q, McAuley J. Dispelling the myth that chronic pain is unresponsive to treatment. British Journal of Sports Medicine 2017; 51: 986988.Scholz J, Mannion RJ, Hord DE, Griffin RS, Rawal B, Zheng H, Scoffings D, Phillips A, Guo J, Laing RJC, Abdi S, Decosterd I, Woolf CJ. A Novel Tool for the Assessment of Pain: Validation in Low Back Pain. PLoS Med 2009;6: e1000047.

Sullivan MJL, Thibault P, Andrikonyte J, Butler H, Catchlove R, Larivière C. Psychological influences on repetition-induced summation of activity-related pain in patients with chronic low back pain. Pain 2009;141: 70-78. 
Sullivan MJL, Thibault P, Savard A, Catchlove R, Kozey J, Stanish WD. The influence of communication goals and physical demands on different dimensions of pain behavior. PAIN 2006; 125: 270-277.

Thomas J, France C, Lavender S, Johnson M. Effects of Fear of Movement on Spine Velocity and Acceleration After Recovery From Low Back Pain. Spine 2008;33: 564-570.

Uddin Z and Macdermid J. Quantitative sensory testing in chronic musculoskeletal pain. . Pain Medicine 2016;17: 1694-1703.

Van Weel C, König-Zahn C, Touw-Otten F, Van Duijn N, Meyboom-de Jong B.Measuring functional status with the COOP/WONCA charts: a manual . 2: UMCG / University of Groningen, Research Institute SHARE.; 2012.

Vibe Fersum K, O'Sullivan P, Skouen JS, Smith A, Kvåle A. Efficacy of classification-based cognitive functional therapy in patients with non-specific chronic low back pain: A randomized controlled trial. European Journal of Pain 2013;17: 916-928.

Vos T and Flaxman AD and Naghavi M and Lozano R and Michaud C and Ezzati M and Shibuya K and Salomon JA and Abdalla S and Aboyans V and Abraham J and Ackerman I and Aggarwal R and Ahn SY and Ali MK and AlMazroa MA and Alvarado M and Anderson HR and Anderson LM and Andrews KG and Atkinson C and Baddour LM and Bahalim AN and Barker-Collo S and Barrero LH and Bartels DH and Basáñez M-G and Baxter A and Bell ML and Benjamin EJ and Bennett D and Bernabé $E$ and Bhalla $K$ and Bhandari B and Bikbov B and Abdulhak AB and Birbeck $\mathrm{G}$ and Black JA and Blencowe $\mathrm{H}$ and Blore JD and Blyth F and Bolliger I and Bonaventure $\mathrm{A}$ and Boufous $\mathrm{S}$ and Bourne $\mathrm{R}$ and Boussinesq $\mathrm{M}$ and Braithwaite $\mathrm{T}$ and Brayne $\mathrm{C}$ and Bridgett $\mathrm{L}$ and Brooker $\mathrm{S}$ and Brooks $\mathrm{P}$ and Brugha TS and Bryan-Hancock $C$ and Bucello $C$ and Buchbinder R and Buckle $G$ and Budke $C M$ and Burch $\mathrm{M}$ and Burney $\mathrm{P}$ and Burstein R and Calabria B and Campbell B and Canter $\mathrm{CE}$ and Carabin $\mathrm{H}$ and Carapetis $\mathrm{J}$ and Carmona $\mathrm{L}$ and Cella $\mathrm{C}$ and Charlson $\mathrm{F}$ and Chen H and Cheng AT-A and Chou D and Chugh SS and Coffeng LE and Colan SD and Colquhoun S and Colson KE and Condon J and Connor MD and Cooper LT and Corriere $\mathrm{M}$ and Cortinovis $\mathrm{M}$ and de Vaccaro $\mathrm{KC}$ and Couser $\mathrm{W}$ and Cowie $\mathrm{BC}$ and Criqui $\mathrm{MH}$ and Cross $\mathrm{M}$ and Dabhadkar KC and Dahiya $\mathrm{M}$ and Dahodwala $\mathrm{N}$ and Damsere-Derry J and Danaei G and Davis A and De Leo D and Degenhardt L and Dellavalle R and Delossantos A and Denenberg J and Derrett S and Des Jarlais DC and Dharmaratne SD and Dherani $\mathrm{M}$ and Diaz-Torne $\mathrm{C}$ and Dolk $\mathrm{H}$ and Dorsey ER and Driscoll T and Duber H and Ebel B and Edmond K and Elbaz A and Ali SE and Erskine $\mathrm{H}$ and Erwin PJ and Espindola $\mathrm{P}$ and Ewoigbokhan SE and Farzadfar F and Feigin $\mathrm{V}$ and Felson DT and Ferrari A and Ferri CP and Fèvre EM and Finucane MM and Flaxman $\mathrm{S}$ and Flood $\mathrm{L}$ and Foreman $\mathrm{K}$ and Forouzanfar $\mathrm{MH}$ and Fowkes FGR and Franklin R and Fransen M and Freeman MK and Gabbe BJ and Gabriel SE and Gakidou E and Ganatra HA and Garcia B and Gaspari F and Gillum RF and Gmel G and Gosselin R and Grainger R and Groeger J and Guillemin F and Gunnell D and Gupta R and Haagsma J and Hagan $\mathrm{H}$ and Halasa YA and Hall W and Haring D and Haro JM and Harrison JE and Havmoeller R and Hay RJ and Higashi $\mathrm{H}$ and Hill C and Hoen B and Hoffman H and Hotez PJ and Hoy D and Huang JJ and Ibeanusi SE and Jacobsen KH and James SL and Jarvis D and Jasrasaria R and Jayaraman S and Johns $\mathrm{N}$ and Jonas JB and Karthikeyan G and Kassebaum N and Kawakami N and Keren A and Khoo J-P and King $\mathrm{CH}$ and Knowlton LM and Kobusingye $\mathrm{O}$ and Koranteng A and Krishnamurthi R and Lalloo R and Laslett LL and Lathlean T and Leasher JL and Lee YY and Leigh J and Lim SS and Limb E and Lin JK and Lipnick $\mathrm{M}$ and Lipshultz SE and Liu W and Loane M and Ohno SL and Lyons R and Ma J and Mabweijano J and MacIntyre MF and Malekzadeh R and Mallinger L and 
Manivannan S and Marcenes W and March L and Margolis DJ and Marks GB and Marks R and Matsumori A and Matzopoulos R and Mayosi BM and McAnulty JH and McDermott MM and McGill N and McGrath J and Medina-Mora ME and Meltzer M and Memish ZA and Mensah GA and Merriman TR and Meyer A-C and Miglioli V and Miller M and Miller TR and Mitchell PB and Mocumbi AO and Moffitt TE and Mokdad AA and Monasta L and Montico M and Moradi-Lakeh M and Moran A and Morawska L and Mori R and Murdoch ME and Mwaniki MK and Naidoo K and Nair MN and Naldi L and Narayan KMV and Nelson PK and Nelson RG and Nevitt MC and Newton CR and Nolte $S$ and Norman P and Norman R and O'Donnell $\mathrm{M}$ and O'Hanlon S and Olives $\mathrm{C}$ and Omer SB and Ortblad $\mathrm{K}$ and Osborne R and Ozgediz D and Page A and Pahari B and Pandian JD and Rivero AP and Patten SB and Pearce N and Padilla RP and Perez-Ruiz F and Perico N and Pesudovs K and Phillips D and Phillips MR and Pierce K and Pion S and Polanczyk GV and Polinder $S$ and Pope Iii CA and Popova S and Porrini E and Pourmalek F and Prince $M$ and Pullan RL and Ramaiah KD and Ranganathan D and Razavi $H$ and Regan $\mathrm{M}$ and Rehm JT and Rein DB and Remuzzi G and Richardson K and Rivara FP and Roberts $\mathrm{T}$ and Robinson $\mathrm{C}$ and De Leòn FR and Ronfani L and Room R and Rosenfeld LC and Rushton L and Sacco RL and Saha S and Sampson U and Sanchez-Riera L and Sanman E and Schwebel DC and Scott JG and Segui-Gomez M and Shahraz S and Shepard DS and Shin H and Shivakoti R and Singh D and Singh GM and Singh JA and Singleton J and Sleet DA and Sliwa K and Smith E and Smith JL and Stapelberg NJC and Steer A and Steiner T and Stolk WA and Stovner LJ and Sudfeld C and Syed $\mathrm{S}$ and Tamburlini G and Tavakkoli $\mathrm{M}$ and Taylor HR and Taylor JA and Taylor WJ and Thomas B and Thomson WM and Thurston GD and Tleyjeh IM and Tonelli M and Towbin JA and Truelsen $\mathrm{T}$ and Tsilimbaris MK and Ubeda $\mathrm{C}$ and Undurraga EA and van der Werf MJ and van Os J and Vavilala MS and Venketasubramanian $\mathrm{N}$ and Wang $\mathrm{M}$ and Wang $\mathrm{W}$ and Watt $\mathrm{K}$ and Weatherall DJ and Weinstock MA and Weintraub R and Weisskopf MG and Weissman MM and White RA and Whiteford $\mathrm{H}$ and Wiersma ST and Wilkinson JD and Williams HC and Williams SRM and Witt E and Wolfe F and Woolf AD and Wulf S and Yeh P-H and Zaidi AKM and Zheng Z-J and Zonies D and Lopez AD and Murray CJL. Years lived with disability (YLDs) for 1160 sequelae of 289 diseases and injuries 1990-2010: a systematic analysis for the Global Burden of Disease Study 2010. The Lancet 2012;380: 2163-2196.

Waddell G, Newton M, Henderson I, Somerville D, Main CJ. A Fear-Avoidance Beliefs Questionnaire (FABQ) and the role of fear-avoidance beliefs in chronic low back pain and disability. PAIN 1993;52: 157-168.

Wai E, Howse K, Pollock W, Dornan H, Vexler L, Dagenais S. The reliability of determining "leg dominant pain"'. The Spine Journal 2009;9: 447-453

Wälti P, Kool J, Luomajoki H. Short-term effect on pain and function of neurophysiological education and sensorimotor retraining compared to usual physiotherapy in patients with chronic or recurrent non-specific low back pain, a piolt randomised controlled trial. BMC Musculoskelet Disorders 2015;16.

Wand B, Catley M, Rabey M, O'Sullivan P, O'Connell N, Smith A. Disrupted selfperception in people with chronic low back pain. Further evaluation of The Fremantle Back Awareness Questionnaire. Journal of Pain 2016;17.

Wong TKT and Lee RYW. Effects of low back pain on the relationship between the movements of the lumbar spine and hip. Human Movement Science 2004;23: 21-34.

Yarnitsky, D., Arendt-Nielsen, L., Bouhassira, D., Edwards, R., Fillingim, R. B., Granot, M., Hansson, P., Lautenbacher, S., Marchand, S. \& Wilder-Smith, O. Recommendations 
on terminology and practice of psychophysical DNIC testing. European Journal of Pain 2010;14: 339. 
Table 1. Descriptive Characteristics of Participants - Total Cohort and Stratified by STarT Back Tool Risk Subgroup

Table 2. Movement Variables - Total Cohort and Stratified by STarT Back Tool Risk Subgroup

Table 3. Sensory Variables - Total Cohort and Stratified by STarT Back Tool Risk Subgroup 


\begin{tabular}{|c|c|c|c|c|c|}
\hline \multirow[b]{2}{*}{ Variable } & \multirow[b]{2}{*}{$\begin{array}{c}\text { Total cohort } \\
n=290 \\
\end{array}$} & \multicolumn{3}{|c|}{ Risk subgroup } & \multirow[b]{2}{*}{ p-value } \\
\hline & & $\begin{array}{c}\text { Low } \\
n=82(28.3 \%)\end{array}$ & $\begin{array}{c}\text { Medium } \\
n=116(40.0 \%)\end{array}$ & $\begin{array}{c}\text { High } \\
n=92(31.7 \%)\end{array}$ & \\
\hline Age (years) & $50.5(37.0-60.0)$ & $49.0(40.0-61.0)$ & $51.5(41.0-58.0)$ & $50.0(32.0-60.0)$ & 0.556 \\
\hline Sex, $n(\%)$ female & $166(57.2)$ & $49(59.8)$ & $73(62.9)$ & $44(47.8)$ & 0.079 \\
\hline Education level (years), mean (SD) & $14.8(3.6)$ & $15.7(3.9)$ & $14.7(3.3)$ & $14.1(3.5)$ & $0.012^{\mathrm{a}}$ \\
\hline Employment status, $n$ (\%) yes working & $223(76.9)$ & $65(79.3)$ & $89(76.7)$ & $69(75.0)$ & 0.799 \\
\hline Occupation, $n(\%)$ & & & & & $<0.001$ \\
\hline Manual & $72(26.2)$ & $13(16.1)$ & $24(22.2)$ & $35(40.7)$ & \\
\hline Sedentary & $174(63.3)$ & $61(75.3)$ & $76(70.4)$ & $37(43.0)$ & \\
\hline Not working & $29(10.6)$ & $7(8.6)$ & $8(7.4)$ & $14(16.3)$ & \\
\hline Compensation status, $n(\%)$ yes compensated & $45(15.8)$ & $15(18.5)$ & $15(13.2)$ & $15(16.9)$ & 0.712 \\
\hline Pain duration (months) & $120.0(42.0-240.0)$ & $120.0(42.0-240.0)$ & $120.0(48.0-300.0)$ & $120.0(36.0-192.0)$ & 0.481 \\
\hline $\begin{array}{l}\text { Overall general health (COOP-WONCA) (lower } \\
\text { scores indicate better health) }\end{array}$ & $3.0(2.0-4.0)$ & $2.0(2.0-3.0)$ & $3.0(2.0-3.5)$ & $3.0(3.0-4.0)$ & $<0.001$ \\
\hline Percent LBP (vs. leg pain), $n(\%)$ & & & & & 0.091 \\
\hline $100 \%$ & $145(50.0)$ & $51(62.2)$ & $49(42.2)$ & $45(48.9)$ & \\
\hline $80 \%$ & $110(38.0)$ & $23(28.1)$ & $50(43.1)$ & $37(40.2)$ & \\
\hline $60 \%$ & $35(12.1)$ & $8(9.8)$ & $17(14.7)$ & $10(10.9)$ & \\
\hline Pain intensity (NRS), mean (SD) & $5.8(1.9)$ & $4.7(1.8)$ & $6.0(1.8)$ & $6.5(1.6)$ & $<0.001$ \\
\hline Disability (RMDQ) & $9.0(6.0-13.0)$ & $6.0(5.0-8.0)$ & $9.0(7.0-13.0)$ & $12.0(8.5-15.0)$ & $<0.001$ \\
\hline
\end{tabular}

$S D=$ standard deviation; NRS = Numerical Rating Scale; RMDQ = Roland Morris Disability Questionnaire; LBP = low back pain. 
Values represent median (interquartile range) unless otherwise indicated. Where there are statistically significant differences between subgroups, group contrasts indicated a significant difference between all three subgroups except where indicated by ${ }^{a}$ (Education level $=$ significant difference between the low and the high risk subgroup only). Boldface indicates statistical significance $(p<0.05)$.

\section{Missing values:}

$n=290$ (total cohort), $n=82$ (low risk subgroup), $n=116$ (medium risk subgroup), and $n=92$ (high risk subgroup) except where there are missing values. Educational level $=14$ missing $(6,1$, and 7 missing respectively from the low, medium, and high risk subgroup).

Occupation $=15$ missing $(1,8$, and 6 missing respectively from the low, medium, and high risk subgroup).

Compensation status $=6$ missing $(1,2$, and 3 missing respectively from the low, medium, and high risk subgroup).

Pain duration $=4$ missing $(0,2$, and 2 missing respectively from the low, medium, and high risk subgroup). 


\begin{tabular}{|c|c|c|c|c|c|c|}
\hline \multirow[b]{2}{*}{ Variable } & \multirow[b]{2}{*}{$\begin{array}{c}\text { Total cohort } \\
n=290\end{array}$} & \multicolumn{3}{|c|}{ Risk subgroup } & \multirow[b]{2}{*}{$\begin{array}{c}\text { Test } \\
\text { statistic }\end{array}$} & \multirow[b]{2}{*}{ p-value } \\
\hline & & $\begin{array}{c}\text { Low } \\
n=82(28.3 \%)\end{array}$ & $\begin{array}{c}\text { Medium } \\
n=116(40.0 \%)\end{array}$ & $\begin{array}{c}\text { High } \\
n=92(31.7 \%)\end{array}$ & & \\
\hline $\begin{array}{l}\text { Pain summation with repeated forward } \\
\text { bending (NRS) }\end{array}$ & $1.0(0.0-2.0)$ & $0.5(0.0-2.0)$ & $1.0(0.0-2.0)$ & $2.0(1.0-3.0)$ & $X^{2}{ }_{(2)}=16.95$ & $<0.001^{\text {ad }}$ \\
\hline $\begin{array}{l}\text { Pain summation with repeated backward } \\
\text { bending (NRS) }\end{array}$ & $0.0(0.0-1.0)$ & $0.0(0.0-1.0)$ & $1.0(0.0-2.0)$ & $0.0(0.0-1.0)$ & $X^{2}(2)=6.90$ & $0.032^{\mathrm{bd}}$ \\
\hline Movement subgroups, $n(\%)$ & & & & & $X^{2}{ }_{(4)}=10.86$ & $0.028^{\mathrm{e}}$ \\
\hline No increase in pain & $143(49.3)$ & $52(63.4)$ & $55(47.4)$ & $36(39.1)$ & & \\
\hline Unidirectional increase in pain & $111(38.3)$ & $24(29.3)$ & $45(38.8)$ & $42(45.7)$ & & \\
\hline Bidirectional increase in pain & $36(12.4)$ & $6(7.3)$ & $16(13.8)$ & $14(15.2)$ & & \\
\hline Forward bending time (seconds) & $18.0(15.0-22.0)$ & $16.0(13.0-20.0)$ & $18.0(15.0-23.0)$ & $19.0(16.0-26.0)$ & $X^{2}{ }_{(2)}=13.48$ & $0.001^{\text {cd }}$ \\
\hline Backward bending time (seconds) & $16.0(14.0-20.0)$ & $15.5(13.0-18.0)$ & $17.0(14.5-21.0)$ & $17.0(14.0-22.0)$ & $X^{2}(2)=10.53$ & $0.005^{c d}$ \\
\hline $\begin{array}{l}\text { Number of protective behaviours per } \\
\text { forward bend completed }\end{array}$ & $1.0(0.4-1.0)$ & $1.0(0.1-1.0)$ & $1.0(0.6-1.0)$ & $1.0(0.6-1.0)$ & $X^{2}(2)=9.67$ & $0.008^{c d}$ \\
\hline $\begin{array}{l}\text { Number of protective behaviours per } \\
\text { backward bend completed }\end{array}$ & $0.0(0.0-0.0)$ & $0.0(0.0-0.0)$ & $0.0(0.0-0.0)$ & $0.0(0.0-0.0)$ & $X^{2}{ }_{(2)}=4.06$ & $0.131^{d}$ \\
\hline
\end{tabular}

NRS = Numerical Rating Scale.

Values represent median (interquartile range). Boldface indicates statistical significance $(p<0.05)$.

\section{Group contrasts:}

${ }^{a}=$ Significant differences between the low and the high risk subgroup and the medium and the high risk subgroup (no significant difference between the low and the medium risk subgroup).

${ }^{b}=$ Significant differences between the low and the medium risk subgroup and the medium and the high risk subgroup (no significant difference between the low and the high risk subgroup). 
${ }^{c}=$ Significant differences between the low and the medium risk subgroup and the low and the high risk subgroup (no significant difference between the medium and the high risk subgroup).

\section{$\mathrm{d}=$ Kruskal-Wallis; ${ }^{\mathrm{e}}=$ chi-squared.}

Missing values:

$n=290$ (total cohort), $n=82$ (low risk subgroup), $n=116$ (medium risk subgroup), and $n=92$ (high risk subgroup) except where there are missing values. Pain summation with repeated forward bending, forward bending time, number of protective behaviours per forward bend = 1 missing (medium risk subgroup).

Pain summation with repeated backward bending, backward bending time, number of protective behaviours per backward bend $=2$ missing (high risk subgroup). 


\begin{tabular}{|c|c|c|c|c|c|c|}
\hline \multirow[b]{2}{*}{ Variable } & \multirow[b]{2}{*}{$\begin{array}{l}\text { Total cohort } \\
\quad n=290\end{array}$} & \multicolumn{3}{|c|}{ Risk subgroup } & \multirow[b]{2}{*}{$\begin{array}{c}\text { Test } \\
\text { statistic }\end{array}$} & \multirow[b]{2}{*}{ p-value } \\
\hline & & $\begin{array}{c}\text { Low } \\
n=82(28.3 \%)\end{array}$ & $\begin{array}{c}\text { Medium } \\
n=116(40.0 \%)\end{array}$ & $\begin{array}{c}\text { High } \\
n=92(31.7 \%)\end{array}$ & & \\
\hline TPD (cm) & $6.0(4.5-7.0)$ & $5.0(4.0-6.0)$ & $6.0(4.5-7.0)$ & $6.0(4.5-7.3)$ & $\mathrm{X}^{2}{ }_{(2)}=8.24$ & $0.016^{\mathrm{ab}}$ \\
\hline TS, $n(\%)$ yes (present) & $54(18.7)$ & $15(18.3)$ & $18(15.7)$ & $21(22.8)$ & $X^{2}{ }_{(2)}=1.74$ & $0.418^{c}$ \\
\hline PPT wrist (kPa) & $269(182-345)$ & $274(189-374)$ & $259(170-365)$ & $276(184-336)$ & $X^{2}(2)=1.07$ & $0.585^{b}$ \\
\hline PPT lumbar (kPa) & $267(163-451)$ & $274(173-484)$ & $242(163-396)$ & $297(156-458)$ & $X^{2}{ }_{(2)}=2.30$ & $0.317^{b}$ \\
\hline CPT wrist $\left({ }^{\circ} \mathrm{C}\right)$ & $5.3(4.0-13.0)$ & $5.5(4.0-11.1)$ & $5.4(4.0-15.4)$ & $4.1(4.0-11.9)$ & $X^{2}{ }_{(2)}=1.40$ & $0.496^{b}$ \\
\hline CPT lumbar $\left({ }^{\circ} \mathrm{C}\right)$ & $4.0(4.0-23.5)$ & $4.0(4.0-17.0)$ & $4.7(4.0-26.0)$ & $4.0(4.0-16.6)$ & $X^{2}(2)=2.69$ & $0.261^{b}$ \\
\hline HPT wrist $\left({ }^{\circ} \mathrm{C}\right)$ & $45.3(42.8-47.8)$ & $44.6(42.1-47.1)$ & $45.2(42.4-48.1)$ & $45.8(43.6-48.3)$ & $X^{2}{ }_{(2)}=2.74$ & $0.254^{b}$ \\
\hline HPT lumbar $\left({ }^{\circ} \mathrm{C}\right)$ & $42.8(40.0-45.6)$ & $42.9(39.7-45.4)$ & $42.4(39.1-45.9)$ & $42.6(40.6-45.8)$ & $X^{2}{ }_{(2)}=0.84$ & $0.658^{b}$ \\
\hline $\begin{array}{l}\text { Baseline CPM pressure to reach } 6 / 10 \text { pain on } \\
\text { NRS ( } \mathrm{kPa})\end{array}$ & $434(260-655)$ & $497(292-651)$ & $398(251-607)$ & $491(248-673)$ & $X^{2}(2)=2.86$ & $0.240^{b}$ \\
\hline CPM change score (NRS), mean (SD) & $1.0(1.3)$ & $0.8(1.3)$ & $0.9(1.1)$ & $1.2(1.4)$ & $F_{(2)}=1.85$ & $0.160^{d}$ \\
\hline
\end{tabular}

TPD = two-point discrimination; $\mathrm{TS}=$ temporal summation; $\mathrm{PPT}=$ pressure pain threshold $; \mathrm{CPT}=$ cold pain threshold; HPT = heat pain threshold; $\mathrm{CPM}=$ conditioned pain modulation; NRS = Numerical Rating Scale; $S D=$ standard deviation.

Values represent median (interquartile range) unless otherwise indicated. Boldface indicates statistical significance $(p<0.05)$.

${ }^{a}=$ Significant differences between the low and the medium risk subgroup and the low and the high risk subgroup (no significant difference between the medium and the high risk subgroup).

${ }^{b}=$ Kruskal-Wallis; ${ }^{c}=$ chi-squared; ${ }^{d}=$ one-way analysis of variance (ANOVA).

Missing values: 
$n=290$ (total cohort), $n=82$ (low risk subgroup), $n=116$ (medium risk subgroup), and $n=92$ (high risk subgroup) except where there are missing values. $\mathrm{TS}=1$ missing (1 missing from the medium risk subgroup).

Baseline CPM pressure, CPM change score $=16$ missing $(6,5$, and 5 missing respectively from the low, medium, and high risk subgroup). 